\title{
Nonlinear peristaltic motion of a Jeffery nanofluid with shear stress and MHD effects
}

\author{
M. Kothandapani*, V Pushparaj**, J. Prakash*** \\ *Department of Mathematics, University College of Engineering Arni (A Constituent College of Anna University Chennai) \\ Arni 632 326, Tamil Nadu, India, Email: mkothandapani@gmail.com \\ **Department of Mathematics, C. Abdul Hakeem College of Engineering \& Technology, Melvisharam 632509 , \\ Tamil Nadu, India, Email: pv_math@yahoo.com \\ ***Department of Mathematics, Agni College of Technology, Thalampur, Chennai 600 130, \\ Tamil Nadu, India, Email: prakashjayavel@yahoo.co.in \\ cross $^{\text {ref }}$ http://dx.doi.org/10.5755/j01.mech.23.3.14481
}

\section{Introduction}

Nowadays, the mechanics of nanofluids has forced the recent researchers for the enhancement of thermal conductivity of base fluid. The word "nanofluid" introduced by Choi [1], it denotes to a liquid suspension containing ultrafine particles having diameter less than 50nm and these particle can be discovered in the metals such as $(\mathrm{Al}, \mathrm{Cu})$, oxides $\left(\mathrm{Al}_{2} \mathrm{O}_{2}\right)$, carbides $(\mathrm{SiC})$, nitrides $(\mathrm{SiN})$ or nonmetals (Graphite, carbon nanotubes, nanofiers, nanosheets, droplets) [2-3]. Nanofluid flows also play indispensable role in some industrial and biomedical instruments, such as drug delivery, photodynamic therapy, molecular motors, neuro electronic interfaces, cancer diagnosis and therapy, surgery, in vivo therapy, neuro electronic interfaces, cell repair machines, protein engineering and shedding new light on cells. It may be famous that particle size is an important physical parameter in nanofluids because it can be used to tailor the nanofluid thermal properties as well as the suspension stability of nanoparticles. The use of magnetic particles in the treatment of cancer is less focused on the delivery of drugs and more on their use as a new therapeutic concept in which tumor cells are damaged by applying local heat through an external magnetic field. A phenomenon that nanofluids has a characteristic feature of thermal conductivity enhancement, which indicates the possibility of using nanofluids in advanced nuclear systems investigated by Masuda et al. [4]. An analytical model for convective transport in nanofluids considering of the Brownian diffusion and thermophoresis are studied by Buongiorno and $\mathrm{Hu}$ [5].

The initial study on the peristaltic flow of a nanofluid in a diverging tube is highlighted by Akbar et al. [6]. They have observed that the pressure rise decreases with the increase in thermophoresis number. Heat transfer and flow field in a wavy channel with nanofluid is numerically examined by Heidary and Kermani [7]. It has been viewed that the skin friction coefficient is almost insensitive to the nanoparticle volume fraction. Akbar and Nadeem [8] considered the peristaltic flow of a nanofluid in an endoscope. The slip effect on the peristaltic flow of nanofluid in an asymmetric channel has been investigated by Akbar et al. [9]. It was observed that the pressure rise decreases when the values of thermophoresis parameter and slip parameter have been increased. Akbar [10] presented the peristaltic flow of a Sisko nanofluid in an asymmetric channel. It can be found that an increase in Sisko nanofluid parameter, pressure rise increases in the peristaltic pumping region. The boundary flow and heat transfer over a permeable stretching sheet due to a nanofluid with the effects of magnetic field, slip boundary condition and thermal radiation were carried out by Ibrahim and Shankar [11]. The influence of nanofluid characteristics on peristaltic heat transfer in a two dimensional axisymmetric channel was discussed by Tripathi and Anwar Beg [12]. They have examined that the nanofluids tend to suppress backflow compared with Newtonian fluids. Akbar et al. [13] studied MHD peristaltic flow of a Carreau nanofluid in an asymmetric channel. Some recent studies of nanofluid due to peristaltic motion are given in Refs. [1421].

Motivated by the above discussion in mind, it is to highlight the significance of the tapered asymmetric channel conditions with peristaltic flow of Jeffery nanofluid. This mathematical model can be viewed as a good application for cervical cancer transport in Cervix blood small vessels. Perhaps, it is very necessary to revise the problem of intrauterine fluid motion in a non-pregnant uterus induced by myometrial contractions which is in the form of a peristaltic type fluid motion and the myometrial contractions may occur in both symmetric and asymmetric directions [22]. To the best of author's knowledge, there is no investigation made yet about the effect of thermal radiation and magnetic field on the peristaltic flow of a Jeffrey nanofluid in the tapered asymmetric channel. The exact analytic solutions for temperature field, nanoparticle fraction field, axial velocity, stream function, pressure gradient and shear stressare obtained under long wavelength and low-Reynolds number assumptions. The effects of various emerging parameters on the flow characteristics are studied in detail with the help of graphs.

\section{Mathematical formulation}

Let us consider a two-dimensional flow of an incompressible Jeffrey nanofluid in a vertical tapered asymmetric channel under the action of a magnetic field. The nanofluid is electrically conducting in the presence of a uniform magnetic field $B_{0}$ applied in the transverse direction. Let $Y=H_{1}$ and $Y=H_{2}$ be right hand side and left 


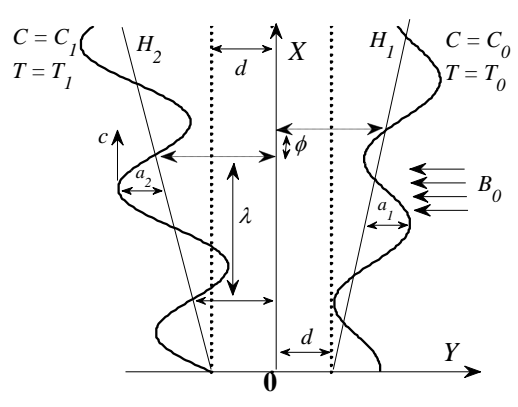

Fig. 1 A physical sketch of the problem

hand side wall boundaries and the medium is considered to be induced by a sinusoidal wave train propagating with a constant speed $c$ along the asymmetric trapped channel wall (Fig. 1) such that:

$$
\begin{aligned}
& H_{1}\left(X, t^{\prime}\right)=d+m^{\prime} X+a_{1} \sin \left[\frac{2 \pi}{\lambda}\left(X-c t^{\prime}\right)\right], \\
& H_{2}\left(X, t^{\prime}\right)=-d-m^{\prime} X-a_{2} \sin \left[\frac{2 \pi}{\lambda}\left(X-c t^{\prime}\right)+\varphi\right] .
\end{aligned}
$$

where $d$ is the half-width of the channel, $a_{1}$ and $a_{2}$ are the amplitudes of left and right walls respectively, $c$ is the phase speed of the wave, $m^{\prime}(<<1)$ is the non - uniform parameter, $\lambda$ is the wave-length, the phase difference $\phi$ varies in the range $0 \leq \phi \leq \pi, \phi=0$ corresponds to symmetric channel with waves out of phase and $\phi=\pi$ the waves are in phase, and further $a_{1}, a_{2} d$ and $\phi$ satisfy the following condition at the inlet of divergent channel, otherwise the walls will be collapsed:

$$
a_{1}^{2}+a_{2}^{2}+2 a_{1} a_{2} \cos (\varphi) \leq(2 d)^{2} .
$$

The constitutive equations for an incompressible Jeffrey nanofluid are:

$$
\begin{aligned}
& T=-p I+S, \\
& S=\frac{\mu}{1+\lambda_{1}}\left(\dot{\gamma}+\lambda_{2} \ddot{\gamma}\right),
\end{aligned}
$$

where $T$ and $S$ are Cauchy stress tensor and extra stress tensor respectively, $p$ is the pressure, $I$ is the identity tensor, $\lambda_{1}$ is the ratio of relaxation to retardation times, $\lambda_{2}$ is the retardation time, $\mu$ is the coefficient of viscosity of the fluid, $\dot{\gamma}$ is the shear rate and dots over the quantities indicate differentiation with respect to time.

The equations governing two-dimensional motion of an incompressible, MHD Jeffrey nanofluid are:

$$
\begin{aligned}
& \rho_{f}\left[\frac{\partial}{\partial t^{\prime}}+U \frac{\partial}{\partial X}+V \frac{\partial}{\partial Y}\right] U=-\frac{\partial p}{\partial X}+\frac{\partial}{\partial X}\left(S_{X X}\right)+ \\
& +\frac{\partial}{\partial Y}\left(S_{X Y}\right)-\sigma^{\prime} B_{0}^{2} U+\left(1-C_{0}\right) \rho_{f} g \alpha\left(T-T_{0}\right)+ \\
& +\left(\rho_{p}-\rho_{f}\right) g \beta^{\prime}\left(C-C_{0}\right),
\end{aligned}
$$

$$
\rho_{f}\left[\frac{\partial}{\partial t^{\prime}}+U \frac{\partial}{\partial X}+V \frac{\partial}{\partial Y}\right] V=-\frac{\partial p}{\partial Y}+\frac{\partial}{\partial X}\left(S_{X Y}\right)+\frac{\partial}{\partial Y}\left(S_{Y Y}\right),
$$

The continuity equation is:

$$
\frac{\partial U}{\partial X}+\frac{\partial V}{\partial Y}=0,
$$

The equation of temperature is:

$$
\begin{aligned}
& \left(\rho c^{\prime}\right)_{f}\left[\frac{\partial T}{\partial t^{\prime}}+U \frac{\partial T}{\partial X}+V \frac{\partial T}{\partial Y}\right]= \\
& =\kappa\left[\frac{\partial^{2} T}{\partial X^{2}}+\frac{\partial^{2} T}{\partial Y^{2}}\right]-\frac{\partial q_{r}}{\partial Y}+\left(\rho c^{\prime}\right)_{p} D_{B}\left(\frac{\partial C}{\partial X} \frac{\partial T}{\partial X}+\frac{\partial C}{\partial Y} \frac{\partial T}{\partial Y}\right)+ \\
& +\frac{D_{T}\left(\rho c^{\prime}\right)_{p}}{T_{m}}\left[\left(\frac{\partial T}{\partial X}\right)^{2}+\left(\frac{\partial T}{\partial Y}\right)^{2}\right],
\end{aligned}
$$

and the nanoparticle volume fraction phenomena is:

$$
\begin{aligned}
& {\left[\frac{\partial C}{\partial t^{\prime}}+U \frac{\partial C}{\partial x}+V \frac{\partial C}{\partial y}\right]=D_{B}\left[\frac{\partial^{2} C}{\partial X^{2}}+\frac{\partial^{2} C}{\partial Y^{2}}\right]+} \\
& +\frac{D_{T}}{T_{m}}\left[\frac{\partial^{2} T}{\partial X^{2}}+\frac{\partial^{2} T}{\partial Y^{2}}\right],
\end{aligned}
$$

where $U, V$ are the components of velocity along $X$ and $Y$ directions respectively, $t^{\prime}$ is the dimensional time, $g$ is the acceleration due to gravity, $P$ is the pressure, $\sigma^{\prime}$ is the electrical conductivity of the fluid, $B_{0}$ is the uniform applied magnetic field, $\rho_{f}$ is the density of the nanofluid, $g$ is the acceleration due to gravity, $\alpha$ the thermal expansion coefficient, $\rho_{p}$ is the density of the nanoparticle, $T_{m}$ is the fluid mean temperature, $c^{\prime}$ is the volumetric volume expansion coefficient, $\kappa$ is the thermal conductivity, $P$ is the pressure, $C$ is the nanoparticle phenomena, the ambient values of $T$ and $C$ as $y$ tend to $H_{1}$ are denoted by $T_{0}$ and $C_{0}$ and $y$ tend to $H_{2}$ are denoted by $T_{1}$ and $C_{1}, D_{B}$ is the Brownian diffusion coefficient, $D_{T}$ is the themophoretic diffusion coefficient $\tau=\frac{\left(\rho c^{\prime}\right)_{p}}{\left(\rho c^{\prime}\right)_{f}}$ is the ratio of the effective heat capacity of nanoparticle material, $\alpha$ is the thermal expansion coefficient, $\beta^{\prime}$ is the coefficient of expansion with concentration and heat capacity of the fluid with $\rho$ being the density. The radiative heat flux in the $X$-direction is considered negligible as compared to $Y$-direction. Hence, by using Rosseland approximation for thermal radiation, the radiative heat flux $q_{r}$ is given by [9]

$$
q_{r}=-\frac{4 \sigma^{*}}{3 k^{*}} \frac{\partial T^{4}}{\partial Y},
$$

where $\sigma *$ and $k *$ are the Stefan-Boltzmann constant and the mean absorption coefficient, respectively. We assume 
that the temperature difference within the flow is sufficiently small such that the term $T^{4}$ in a Taylor series about a free stream temperature $T_{0}$ and Neglecting higher order terms in the first order in $\left(T-T_{0}\right)$, we obtain:

$$
T^{4} \cong 4 T_{0}^{3} T-3 T_{0}^{4}
$$

Thus, substituting the Eq. (9) into Eq. (10), we get:

$$
q_{r}=-\frac{16 \sigma * T_{0}^{3}}{3 k *} \frac{\partial T}{\partial Y}
$$

In order to describe the fluid flow in a non-dimensional form, we introduce following quantities in Eqs. (5,7$11)$,

$$
\begin{aligned}
& x=\frac{X}{\lambda}, y=\frac{Y}{d}, t=\frac{c t^{\prime}}{\lambda}, u=\frac{U}{c}, v=\frac{V}{c}, \delta=\frac{d}{\lambda}, h_{1}=\frac{H_{1}}{d}, \\
& h_{2}=\frac{H_{2}}{d}, p=\frac{d^{2} P}{c \lambda \mu}, \theta=\frac{T-T_{0}}{T_{1}-T_{0}}, S c=\frac{v}{D_{B}}, \\
& a=\frac{a_{1}}{d}, b=\frac{a_{2}}{d}, m=\frac{\lambda m^{\prime}}{d}, \sigma=\frac{C-C_{0}}{C_{1}-C_{0}}, R=\frac{\rho_{f} c d}{\mu}, \\
& G_{r}=\frac{\left(1-C_{0}\right) \rho_{f} g \alpha d^{2}\left(T_{1}-T_{0}\right)}{c \mu}, B_{r}=\frac{\rho g \beta^{\prime} d^{2}\left(C_{1}-C_{0}\right)}{c \mu} \\
& s=\frac{d}{\mu c} S, P_{r}=\frac{\mu c_{f}}{\kappa}, N_{b}=\frac{\tau D_{B}\left(C_{1}-C_{0}\right)}{v}, \\
& N_{t}=\frac{\tau D_{T}\left(T_{1}-T_{0}\right)}{T_{m} v}, M=\sqrt{\frac{\sigma^{\prime}}{\mu}} d B_{0}, R_{n}=\frac{4 \sigma^{*} T_{0}^{3}}{3 k^{*} \mu c^{\prime}} .
\end{aligned}
$$

and the stream function $u=\frac{\partial \psi}{\partial y}, v=-\delta \frac{\partial \psi}{\partial x}$.

Using the long wavelength approximation and neglecting the wave number along with low-Reynolds number, one can find from Eqs. (4-8) that:

$$
\begin{aligned}
& \frac{\partial p}{\partial x}=\frac{\partial}{\partial y}\left(\frac{1}{1+\lambda_{1}} \frac{\partial^{2} \psi}{\partial y^{2}}\right)-M^{2} \frac{\partial \psi}{\partial y}+G_{r} \theta+B_{r} \sigma, \\
& \frac{\partial p}{\partial y}=0, \\
& \left(\frac{3+4 R n \operatorname{Pr}}{3 \operatorname{Pr}}\right) \frac{\partial^{2} \theta}{\partial y^{2}}+N b\left(\frac{\partial \sigma}{\partial y} \frac{\partial \theta}{\partial y}\right)+N t\left(\frac{\partial \theta}{\partial y}\right)^{2}=0, \\
& \frac{\partial^{2} \sigma}{\partial y^{2}}+\frac{N t}{N b} \frac{\partial^{2} \theta}{\partial y^{2}}=0 .
\end{aligned}
$$

Elimination of pressure from Eqs. (13) and (14), gives:

$$
\frac{\partial^{2}}{\partial y^{2}}\left(\frac{1}{1+\lambda_{1}} \frac{\partial^{2} \psi}{\partial y^{2}}\right)-M^{2} \frac{\partial^{2} \psi}{\partial y^{2}}+G r \frac{\partial \theta}{\partial y}+B r \frac{\partial \sigma}{\partial y}=0 .
$$

The corresponding boundary conditions are given below:

$$
\begin{aligned}
& \psi=\frac{F}{2}, \frac{\partial \psi}{\partial y}=0, \theta=1 \text { and } \sigma=1 \text { at } \\
& y=h_{1}=1+m x+a \sin (2 \pi(x-t)), \\
& \psi=-\frac{F}{2}, \frac{\partial \psi}{\partial y}=0, \theta=0 \text { and } \sigma=0 \text { at } \\
& y=h_{2}=-1-m x-b \sin (2 \pi(x-t)+\varphi) .
\end{aligned}
$$

where $x$ is non-dimensional axial coordinate, $y$ is non-dimensional transverse coordinate, $t$ is dimensionless time, $u$ and $v$ are non-dimensional axial and transverse velocity components, $p$ is dimensionless pressure, $a$ and $b$ are amplitudes of right and left walls respectively, $S c$ is the Schmidt number, $\delta$ is wave number, $m$ is the non - uniform parameter, $R$ is the Reynolds number, $v$ is the nanofluid kinematic viscosity, $\theta$ is the dimensionless temperature, $\sigma$ is the dimensionless rescaled nanoparticle volume fraction, $P_{r}$ is the Prandtl number, $M$ is the Hartmann number, $G_{r}$ is the local temperature Grashof number, $B_{r}$ is the local nanoparticle Grashof number, $N_{b}$ is the Brownian motion parameter, $N_{t}$ is the thermophoresis parameter and $R_{n}$ is the thermal radiation parameter.

The average flow rate defined as $[15-16,25]$ :

$$
F(x, t)=\Theta+a \sin 2 \pi(x-t)+b \sin [2 \pi(x-t)+\varphi]
$$

in which

$$
F=\int_{h_{1}}^{h_{2}} u d y
$$

\section{Exact Solution of the problem}

Integration of Eq. (16) with respect to $y$ and implementation in Eq. (15) and boundary conditions of Eqs. (18), the dimensionless temperature field is obtained as:

$$
\theta=e^{A_{1}\left(h_{2}-y\right)}\left(\frac{1-e^{A_{1}\left(y-h_{1}\right)}}{1-e^{A_{1}\left(h_{2}-h_{1}\right)}}\right),
$$

substituting Eq. (20) into Eq. (16), moreover Eq. (16) integrating with respect to $y$ and using proper boundary conditions of Eq. (18), the nanoparticle fraction field is received as:

$$
\sigma=\frac{\left(h_{1}-y\right)\left(N_{b}+N_{t}\right)}{N_{b}\left(h_{1}-h_{2}\right)}+\frac{N_{t} e^{A_{1}\left(h_{2}-h_{1}\right)}}{N_{b}}\left(\frac{1-e^{A_{1}\left(h_{1}-y\right)}}{1-e^{A_{1}\left(h_{2}-h_{1}\right)}}\right),
$$

differentiation of Eqs. (20-21) with respect to $y$, then substituting in Eq. (17), we get:

$$
\begin{aligned}
& \psi=A_{2}+A_{3} y+A_{4} \cosh \left(M \sqrt{1-\lambda_{1}} y\right)+ \\
& +A_{5} \sinh \left(M \sqrt{1-\lambda_{1}} y\right)+A_{6} e^{-A_{1} y}+A_{7} y^{2},
\end{aligned}
$$




$$
\begin{gathered}
u=A_{3}+M \sqrt{1-\lambda_{1}}\left(\begin{array}{l}
A_{4} \sinh \left(M \sqrt{1-\lambda_{1}} y\right)+ \\
+A_{5} \cosh \left(M \sqrt{1-\lambda_{1}} y\right)
\end{array}\right)- \\
-A_{1} A_{6} e^{-A_{1} y}+2 A_{7} y \\
\frac{\partial p}{\partial x}=-\frac{\left(A_{1}^{3}-\left(1+\lambda_{1}\right) A_{1}\right) A_{6}}{1+\lambda_{1}}-\frac{h_{1} B_{r}\left(1+\lambda_{1}\right)\left(N_{b}-N_{t}\right)}{N_{b}\left(h_{2}-h_{1}\right)}- \\
-A_{3} N^{2}+\frac{\left(e^{-A_{1} h_{1}}-1\right)\left(1+\lambda_{1}\right)\left(N_{t} \cdot B_{r}-N_{b} \cdot G_{r}\right)}{N_{b}\left(e^{-A_{1} h_{2}}-e^{-A_{1} h_{1}}\right)} .
\end{gathered}
$$

The average rise in pressure $\Delta P_{\lambda}$ is evaluated by taking the average values of $\Delta P_{\lambda}(t)$ over one period of wave are given as follows:

$$
\Delta P_{\lambda}=\int_{0}^{1} \int_{0}^{1}\left(\frac{\partial p}{\partial x}\right)_{y=0} d x d t
$$

the non-dimensional shear stress at the left wall of the channel is reduced to:

$$
S_{x y}=\frac{1}{1+\lambda_{1}} \frac{\partial^{2} \psi}{\partial y^{2}}=\frac{1}{\left(1+\lambda_{1}\right)}\left(\begin{array}{l}
2 A_{7}+A_{4} N^{2} \cosh \left(N h_{2}\right)+ \\
+A_{5} N^{2} \sinh \left(N h_{2}\right)+A_{1}^{2} A_{6} e^{-A_{1} h_{2}}
\end{array}\right),
$$

where

$$
\begin{aligned}
& N=M \sqrt{1-\lambda_{1}} \text {, } \\
& A_{13}=A_{6}\left(e^{-A_{1} h_{2}}-e^{-A_{1} h_{1}}+A_{1} A_{6}\left(h_{2}-h_{1}\right) e^{-A_{1} h_{2}}\right)-A_{7}\left(h_{2}-h_{1}\right)^{2}-F, \\
& A_{1}=\frac{3 P_{r}\left(N_{t}+N_{b}\right)}{\left(h_{2}-h_{1}\right)\left(3+4 P_{r} \cdot R_{n}\right)} \text {. } \\
& A_{12}=\sinh \left(N h_{2}\right)-\sinh \left(N h_{1}\right)-N\left(h_{2}-h_{1}\right) \cosh \left(N h_{2}\right) \text {, } \\
& A_{11}=\cosh \left(N h_{2}\right)-\cosh \left(N h_{1}\right)-N\left(h_{2}-h_{1}\right) \sinh \left(N h_{2}\right) \text {, } \\
& A_{10}=2 A_{7}\left(h_{2}-h_{1}\right)-A_{1} A_{6}\left(e^{-A_{1} h_{2}}-e^{-A_{1} h_{1}}\right) \text {, } \\
& A_{9}=N\left(\cosh \left(N h_{2}\right)-\cosh \left(N h_{1}\right)\right) \text {, } \\
& A_{8}=N\left(\sinh \left(N h_{2}\right)-\sinh \left(N h_{1}\right)\right), A_{7}=\frac{B_{r}\left(1+\lambda_{1}\right)\left(N_{b}+N_{t}\right)}{2 N^{2} N_{b}\left(h_{2}-h_{1}\right)}, \\
& A_{6}=\frac{\left(G_{r} \cdot N_{b}-B_{r} \cdot N_{t}\right)\left(1+\lambda_{1}\right)}{N_{b}\left(e^{-A_{1} h_{2}}-e^{-A_{1} h_{1}}\right)\left(A_{1}^{3}-N^{2} A_{1}\right)}, A_{5}=\frac{A_{8} A_{13}-A_{10} A_{11}}{A_{9} A_{11}-A_{8} A_{12}}, \\
& A_{4}=-\frac{1}{A_{8}}\left(A_{9} A_{5}+A_{10}\right) \text {, } \\
& A_{3}=-N B_{4} \sinh \left(N h_{2}\right)-N B_{5} \cosh \left(N h_{2}\right)+A_{1} e^{-A h_{2}}-2 A_{7} h_{2}, \\
& A_{2}=\frac{F}{2}-A_{3} h_{2}-A_{4} \cosh \left(N h_{2}\right)-A_{5} \sinh \left(N h_{2}\right)- \\
& -A_{6} e^{-A h_{2}}-A_{7} h_{2}^{2} \text {. }
\end{aligned}
$$

\section{Numerical results and discussion}

In this section, numerical effects of the problem under discussion are investigated by graphs. The expression for average pressure rise is computed numerically using mathematical softwares Mathematica and Matlab. Figs. (24) are exposed to see the influences of average rise in pressure for various values of the non-uniform parameter $(m)$, Hartmann number $(M)$, Jeffrey parameter $\left(\lambda_{1}\right)$. The graph is sectored in four parts (i.e.) the upper right- hand quadrant (I) denotes the region of the peristaltic pumping ( $\Theta>0$ and $\Delta p_{\lambda}>0$ ). Quadrant (II) is designated as augmented flow when $\Theta>0$ and $\Delta p_{\lambda}<0$. Quadrant (IV) such that $\Theta<0$ and $\Delta p_{\lambda}>0$ is called retrograde or backward pumping. It indicates that there is a linear relation between $\Delta p_{\lambda}$ and $\Theta$ . It is found from Fig. 2 that with an increase in non-uniform parameter $m$, the average rise in pressure decreases in peristaltic pumping. Fig. 3 gives the influences of Hartmann number $M$ on $\Delta p_{\lambda}$, it is examined that an increase in $M$ results decrease in the peristaltic pumping rate, free pumping and adverse pressure gradient. The variation of average rise in pressure with the mean flow for different values of $\lambda_{1}$ is shown in Fig.4. It is clear that the average rise in pressure increases when $\lambda_{1}$ increases.

Velocity profiles are plotted in Figs. (5-6) to study the effects of different parameters such as $\lambda_{1}$ and $M$. It is

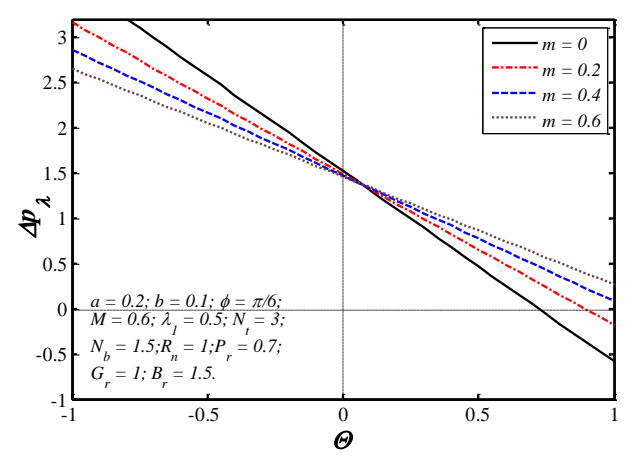

Fig. 2 Variation of pressure rise $\Delta p_{\lambda}$ versus $\Theta$ for different values of $m$

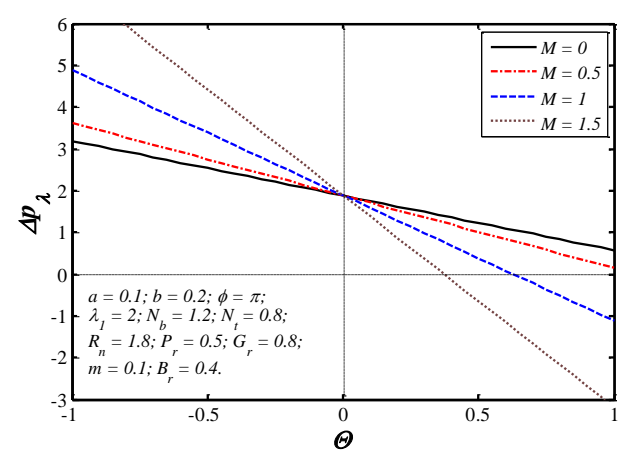

Fig. 3 Variation of pressure rise $\Delta p_{\lambda}$ versus $\Theta$ for different values of $M$

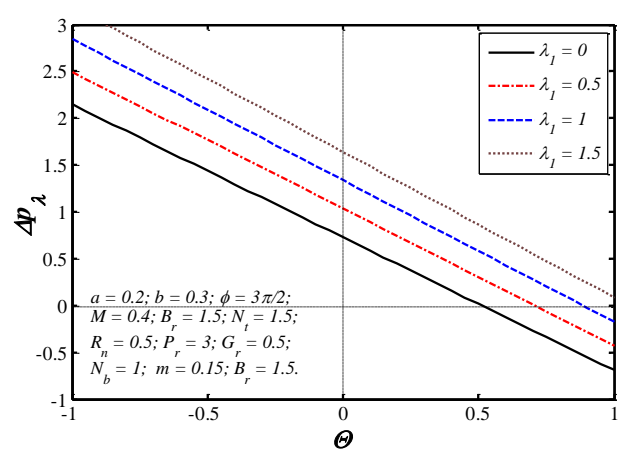

Fig. 4 Variation of pressure rise $\Delta p_{\lambda}$ versus $\Theta$ for different values of $M$ 


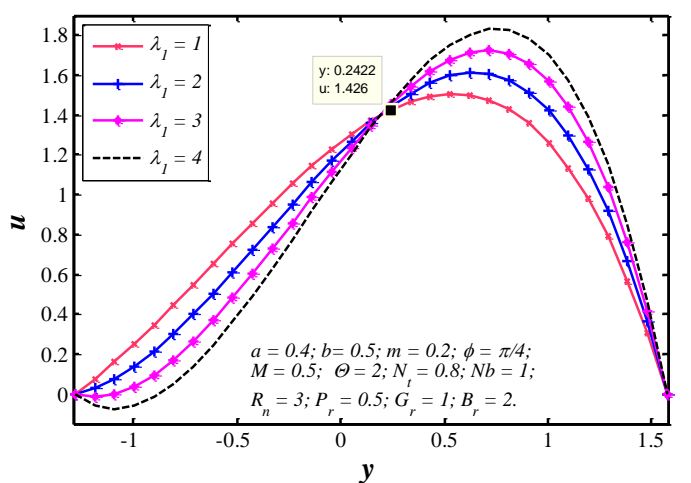

Fig. 5 Influence of $\lambda_{1}$ on axial velocity $(u)$

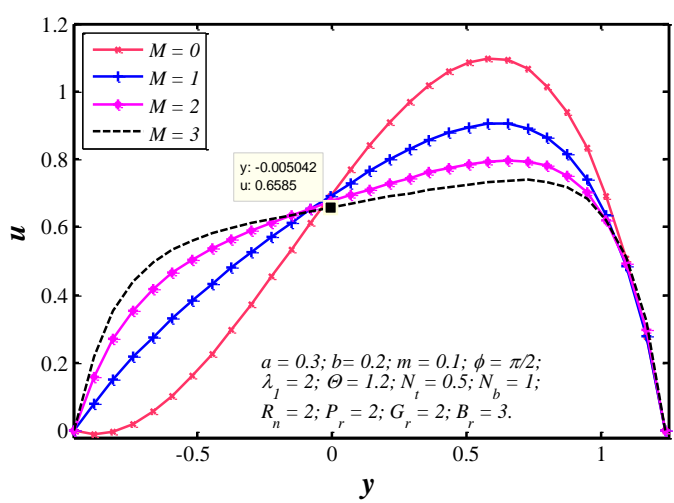

Fig. 6 Influence of $M$ on axial velocity $(u)$

the maximum velocities are always occurred at the heart part of the channel, decaying smoothly to zero at the periphery (channel wall). Fig. 5 is drawn to study the effect of Jeffrey parameter $\left(\lambda_{1}\right)$ on the axial velocity $(u)$. It reveals that the velocity profiles are parabolic. Moreover, the axial velocity decreases in the region $y \in[-1.42,0.24]$ and in other part of the channel it increases as Jeffrey parameter increases. The axial velocity for the Hartmann number $M$ is illustrated in Fig. 6. It is observed that an increase in $M$ causes decrease in magnitude of axial velocity $u$ at the centre part of the channel. Physically speaking, Magnetic nanoparticles were injected into the tumor and then heated in an alternating magnetic field. The instillation of magnetic nanoparticles in glioblastoma multiforme (GBM) patients induced the uptake of nanoparticles in macrophages to a major extent, and the uptake was further promoted by magnetic fluid hyperthermia (MFH) therapy [23].

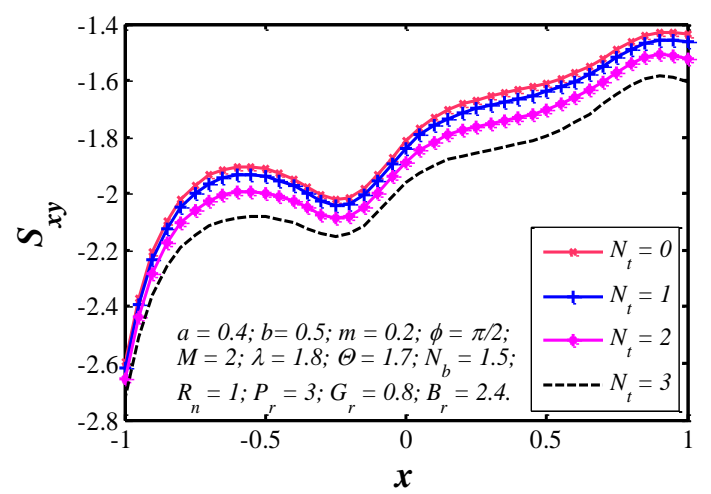

Fig. 7 The axial shear stress distributions at the upper wall for different value $N_{t}$

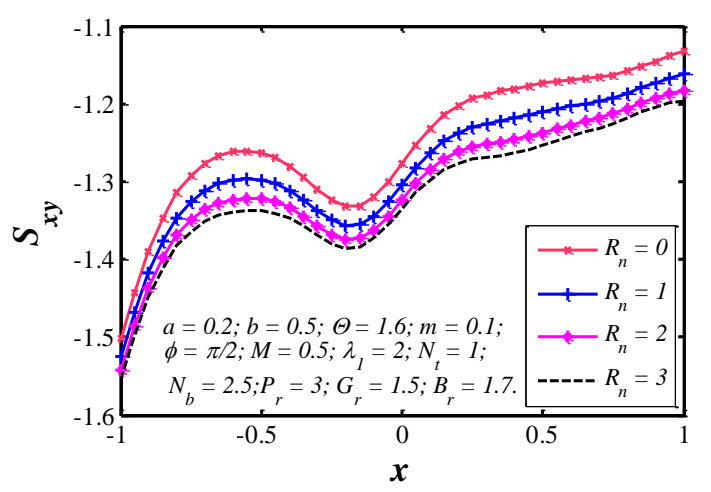

Fig. 8 The axial shear stress distributions at the upper wall for different value $R_{n}$

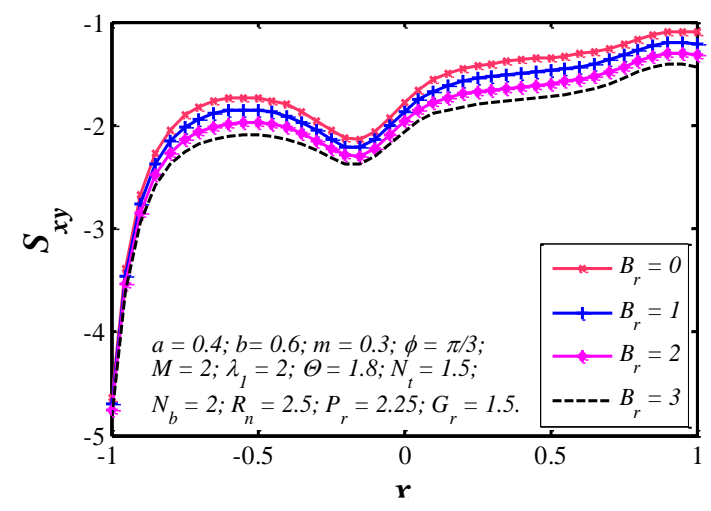

Fig. 9 The axial shear stress distributions at the upper wall for different value $B_{r}$

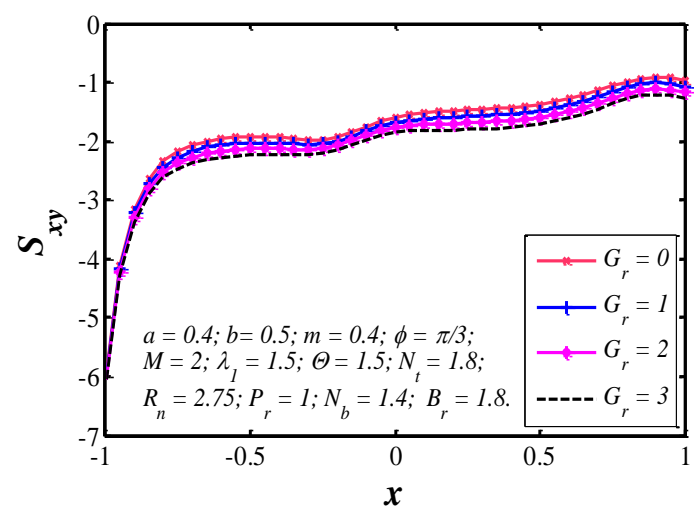

Fig. 10 The axial shear stress distributions at the upper wall for different value $G_{r}$

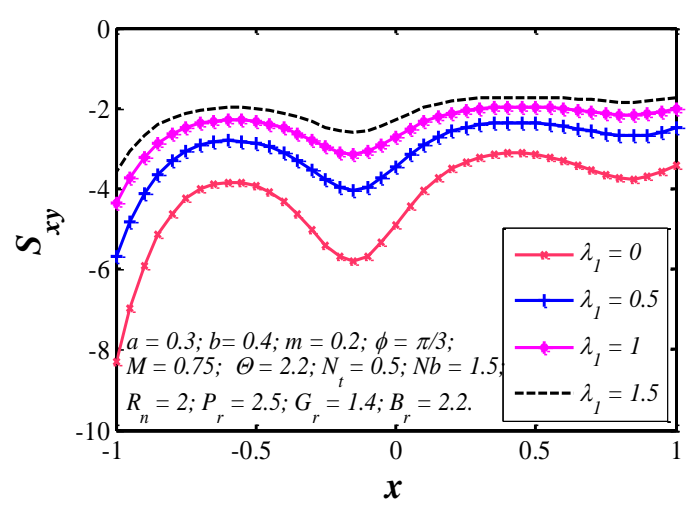

Fig. 11 The axial shear stress distributions at the upper wall for different value $\lambda_{1}$ 


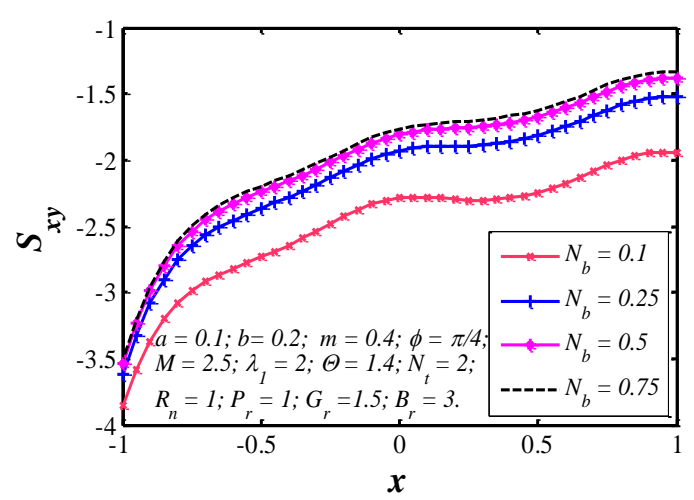

Fig. 12 The axial shear stress distributions at the upper wall for different value $N_{b}$

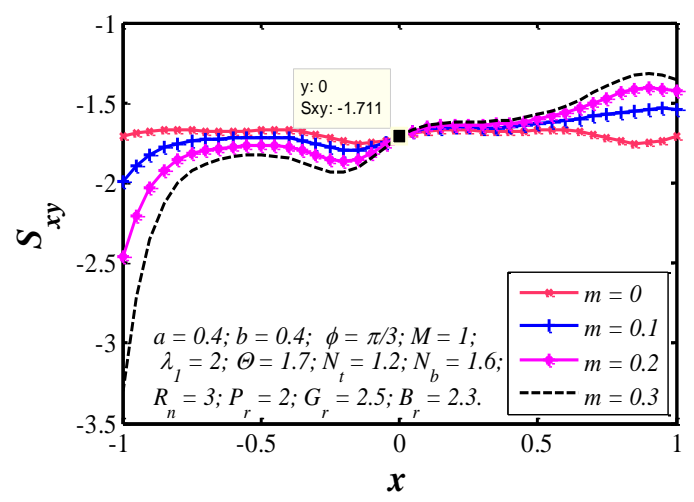

Fig. 13 The axial shear stress distributions at the upper wall for different value $m$
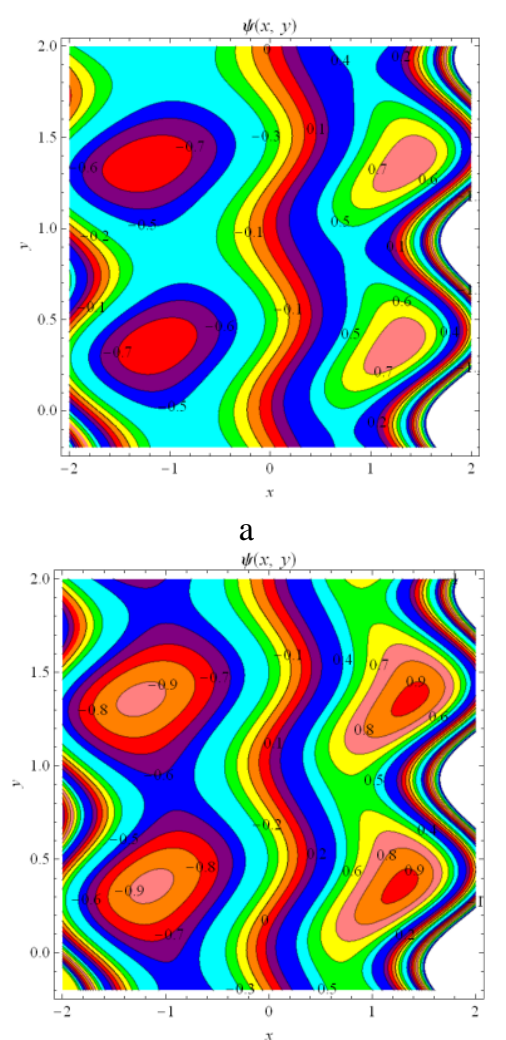

$\mathrm{b}$

Fig. 14 Streamlines when $a=0.2, \quad b=0.3, \quad m=0.1$,

$$
\begin{aligned}
& \phi=\pi / 2, \quad M=1, \lambda_{1}=3, \quad N_{t}=0.8, \quad N_{b}=0.5, \\
& R_{n}=0.5, \quad P_{r}=1, \quad G_{r}=1, \quad B_{r}=1, \quad t=0.2 \\
& \Theta=1.2,(\mathrm{~b}) \Theta=1.5
\end{aligned}
$$

The axial shear stress distribution $\left(S_{x y}\right)$ on the right wall of the tapered asymmetric channel is presented in Figs. (7-13). Physically speaking, the particles settled rapidly, forming a layer on the surface and reducing the heat transfer capacity of the fluid. We notice that stress is in oscillatory behaviour, which may due to peristalsis. Figs. (7-12) demonstrate that the effects of $N_{t}, R_{n}, B_{r}, G_{r}, \lambda_{1}$ and $N_{b}$ on the axial shear stress. It is observed that the amplitude of the stress oscillations decreases as $N_{t}, R_{n}, B_{r}$ and $G_{r}$, while absolute value of axial shear stress increases as $\lambda_{1}$ and $N_{b}$ increase. Moreover, the values of axial shear stress are larger in case of a Jeffrey fluid when compared with Newtonian fluid. In Fig. 13 the axial shear stress $S_{x y}$ is graphed versus $y$ for different values of $m$. We can notice that, when $x<0$ shear stress decreases with increasing $m$ but this behavior is reversed, when $x>0$.

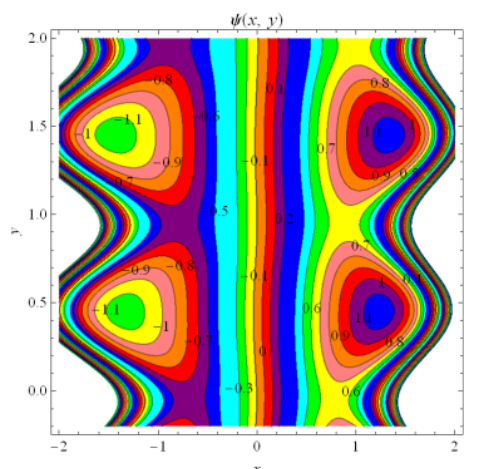

a

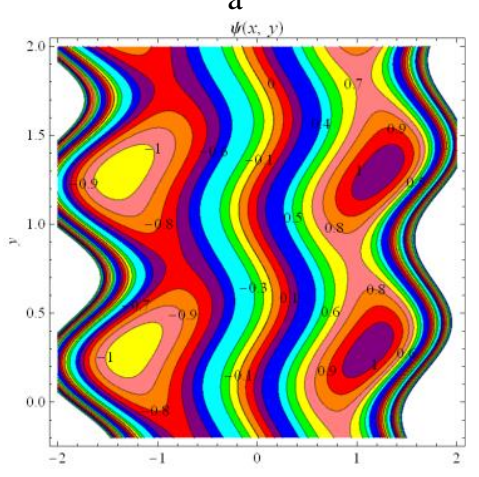

b

Fig. 15 Streamlines when $a=0.3, b=0.2, m=0.1$,

$$
\begin{aligned}
& \Theta=1.8, \quad M=2, \quad \lambda_{1}=1, \quad N_{t}=1, \quad N_{b}=3, \quad R_{n}=2, \\
& P_{r}=2, G_{r}=1.5, \quad B_{r}=1.5, t=0.2 \quad \text { (a) } \phi=0, \quad \text { (b) } \\
& \phi=\pi / 2
\end{aligned}
$$

Another inspiring phenomena of peristalsis is trapping, the expression of an internally circulating bolus of fluid which moves along with the wave. Based on the effects of the mean flow rate $(\Theta)$ on trapping, we have prepared Fig. 14. We interest to observe that the trapping also exists along the right and left walls of the tapered asymmetric channel. The results show that the trapped bolus increases along both the walls with increasing $\Theta$. The streamlines for the different values of phase difference $\phi$ are plotted in Figs.15. It is observed that the number and size of the trapped bolus decrease with the increase in $\phi$. 


\section{Concluding remarks}

In the present study, an analysis of peristaltic motion of a Jeffrey nanofluid in the tapered asymmetric channel under a uniform magnitude field has been made. The governing two dimensional equations have been modelled and then simplified using long wavelength and low - Reynolds number approximations. Exact expression of temperature field, nanoparticle fraction field, axial velocity, stream function, pressure gradient, shear stress, heat and nanoparticle volume fraction transfer coefficients are developed. The results are discussed through graphs. We conclude the following observations:

- It is found that the average rise in pressure increases when the non-uniform parameter $(m)$ and Jeffrey parameter $\left(\lambda_{1}\right)$ are increased.

- The axial velocity increases with increasing $\lambda_{1}$ while it decreases with increasing $M$ in the core part of the channel.

- It is analyzed amplitude of the stress oscillations decreases as $R_{n}$ and $G_{r}$, while absolute value of axial shear stress increases as $\lambda_{1}$ and $N_{b}$ increase.

- The size of the trapped bolus decreases with an increase in phase difference.

- Further it has also been noticed that the size of the trapped bolus decreases with increasing $\lambda_{1}$. When amplitude of $a=b$, phase difference $\phi=0$, Prandtl number $P_{r}=1$, radiation parameter $R_{n}=0$, rheological parameter $\lambda_{1}=0$ and non-uniform parameter $m=0$, our results are in good agreement with Tripathi and Anwar Beg [24].

\section{References}

1. Choi S.U.S. 1995. Enhancing thermal conductivity of fluids with nano-particles, ASME Fluids Engineering Division 231:99-103.

2. HabibiMatin, M.; Dehsara, M.; Abbassi, A. 2012. Mixed convection MHD flow of nanofluid over a nonlinear stretching sheet with effects of viscous dissipation and variable magnetic field, Mechanika 18(4): 415-423. http://dx.doi.org/10.5755/j01.mech.18.4.2334.

3. HabibiMatin, M.; Hosseini, R.; Simiari, M.; Jahangiri, P. 2013. Entropy generation minimization of nanofluid flow in a MHD channel considering thermal radiation effect, Mechanika 19(4): 445-450. http://dx.doi.org/10.5755/j01.mech.19.4.5050.

4. Masuda, H.; Ebata, A.; Teramae, K.; Hishinuma, N. 1993. Alteration of thermal conductivity and viscosity of liquid by dispersing ultra-fine particles, NetsuBussei 7: 227-233. http://dx.doi.org/10.2963/jjtp.7.227.

5. Buongiorno, J. 2006. Convective transport in nanofluids, ASME Journal of Heat Transfer 128: 240-250. http://dx.doi.org/10.1115/1.2150834.

6. Akbar, N.S.;Nadeem, S.;Hayat, T.;Hendi, A.A. 2012. Peristaltic flow of a nanofluid in a non-uniform tube, Heat and Mass Transfer 48: 451- 459. http://dx.doi.org/10.1007/s00231-011-0892-7.
7. Heidary, H.; Kermani, K.J. 2010. Effect of nano particles on forced convection in sinusoidal wall channel, International Communication om Heatand Mass transfer 37: 1520-1527.

http://dx.doi.org/10.1016/j.icheatmasstransfer. 2010.08.018.

8. Akbar, N.S.; Nadeem, S. 2011. Endoscopic effects on peristaltic flow of a nanofluid, Communications in Theoretical Physics 56: 761-768.

http://iopscience.iop.org/0253-6102/56/4/28.

9. Akbar, N.S.; Nadeem, S.; Hayat, T.; Hendi, A. 2012. Peristaltic flow of a nanofluid with slip effects, Meccanica 47: 1283-1294.

http://dx.doi.org/10.1007/s11012-011-9512-3.

10. Akbar, N.S. 2013. Peristaltic Siskonanofluid in an asymmetric channel, Applied Nanoscience 4: 663-673. http://dx.doi.org/10.1007/s13204-013-0205-1.

11. Ibrahim, W.; Shankar, B. 2013. MHD boundary layer flow and heat transfer of a nanofluid past a permeable stretching sheet with velocity, thermal and solutal slip boundary conditions, Computer and Fluids 75: 1-10. http://dx.doi.org/10.1016/j.compfluid.2013.01.014.

12. Tripathi, D.; Beg, A.O. 2014. A study on Peristaltic flow of nanofluids: Application in drug delivery systems, International Journal of Heat and Mass Transfer 70: 61-70.

http://dx.doi.org/10.1016/j.ijheatmasstransfer. 2013.10.044.

13. Akbar, N.S.; Nadeem, S.; Khan, Z.H. 2013. Numerical simulation of peristaltic flow of a Carreaunanofluid in an asymmetric channel, Alexandria Engineering Journal 53: 191-197.

http://dx.doi.org/10.1016/j.aej.2013.10.003.

14. Noreen, S. 2014. Mixed convection peristaltic flow with slip condition and induced magnetic field, Eur. Phys. J. Plus 129: 33.

http://dx.doi.org/10.1140/epjp/i2014-14033-3.

15. Kothandapani, M.; Prakash, J. 2015. Effects of thermal radiation parameter and magnetic field on the peristaltic motion of Williamson nanofluids in a tapered asymmetric channel, International Journal of Heat and Mass Transfer81: 234-245.

http://dx.doi.org/10.1016/j.ijheatmasstransfer. 2014.09.062.

16. Kothandapani, M.; Prakash, J. 2015. The peristaltic transport of CarreauNanofluids under effect of a magnetic field in a tapered asymmetric channel: Application of the cancer therapy, Journal of Mechanics in Medicine and Biology 15: 1550030. http://dx.doi.org/10.1142/S021951941550030X.

17. Aly, E.H.; Ebaid, A. 2014. Exact analytical solution for the peristaltic flow of nanofluids in an asymmetric channel with slip effect of the velocity, temperature and concentration, Journal of Mechanics 30: 411-422. http://dx.doi.org/10.1017/jmech.2014.13.

18. Akbar, N.S. 2014. Double-diffusive natural convective peristaltic flow of a Jeffrey nanofluid in a porous channel, Heat Transfer Research 45: 293-307. http://dx.doi.org/10.615/HeatTransRes.2013006995.

19. Hayat, T.; Abbasi , F.M.; Al-Yami, M.; Monaquel, S. 2014. Slip and Joule heating effects in mixed convection peristaltic transport of nanofluid with Soret and Dufour effects, Journal of Molecular Liquids 194: 93-99. 
http://dx.doi.org/10.1016/j.molliq.2014.01.021.

20. Ellahi, R.; Riaz, A.; Nadeem, S. 2014. A theoretical study of Prandtlnanofluid in a rectangular duct through peristaltic transport, Applied Nanoscience 4: 753-760 http://dx.doi.org/10.1007/s13204-013-0255-4.

21. Nadeem, S.; Riaz, A.; Ellahi, R.; Akbar, N.S. 2014. Effects of heat and mass transfer on peristaltic flow of a nanofluid between eccentric cylinders, Applied Nanoscience 4: 393-404. http://dx.doi.org/10.1007/s13204-013-0225-x.

22. Eytan, O.; Jaffa, A.J.; Elad, D. 2001. Peristaltic flow in a tapered channel: application to embryo transport within the uterine cavity, Medical Engineering \& Physics 23: 473-484. http://dx.org/10.1016/S1350-4533(01)00078-9.

23. Van Landeghem, F.K.H.; Maier-Hauff, K.; Jordan, A.; Hoffmann, K.T.; Gneveckow, U.; Scholz, R.; Thiesen, B.; Bruck, W.; Von Deimling, A. 2009. Postmortem studies in glioblastoma patients treated with thermotherapy using magnetic nanoparticles, Biomaterials 30: 52-57. http://dx.doi.org/10.1016/j.biomaterials.2008.09.044.

24. Tripathi, D.; Beg, A.O. 2014. A study on Peristaltic flow of nanofluids: Application in drug delivery systems, International Journal of Heat and Mass Transfer 70: $61-70$ http://dx.doi.org/10.1016/j.ijheatmasstransfer. 2013.10.044.

25. Srivastava, L.M.; Srivastava, V.P.; Sinha, S.N. 1983. Peristaltic transport of a physiological fluid: Part I. flow in non-uniform geometry, Biorheology 20: 153-166.
M. Kothandapani, V Pushparaj, J. Prakash

\section{NONLINEAR PERISTALTIC MOTION OF A JEFFERY NANOFLUID WITH SHEAR STRESS AND MHD EFFECTS}

S u m m a r y

A mathematical model based on Jeffrey nanofluid under the effect of magnetic field and thermal radiation parameter for the peristaltic flow is considered in a channel, which is assumed to be in the form of a tapered asymmetric walls. The tapered asymmetry channel is produced by choosing the peristaltic wave train on the non-uniform walls to have different amplitudes and phase but with same wave speed. The analytical expressions for temperature field, nanoparticle fraction field, axial velocity, stream function, pressure gradient and shear stress are derived under the assumptions of long wavelength and low Reynolds number approximations. The salient characteristics of pumping and trapping are discussed with particular focus on the effect of geometry parameters, Hartmann number, thermal radiation parameter and rheological parameter. It has been observed that the pressure rise and axial velocity increase with increasing rheological parameter $\lambda_{1}$.

Keywords: Peristaltic transport; Jeffrey Nanofluid; Shear stress; MHD; Tapered asymmetric channel.

Received April 08, 2016

Accepted June 08, 2017 